\title{
Enough is Enough? The Changing World of Ischemic Stroke Workup
}

\author{
S. Andrew Josephson, MD*
}

Department of Neurology, University of California, San Francisco, San Francisco, California.

After entertaining the possibility of acute intervention, the majority of hospitalists' efforts in the management of patients with ischemic stroke involve identifying an etiology and initiating secondary prevention strategies. Other than evaluating stroke risk factors, workup has traditionally involved extracranial and intracranial vessel imaging, cardiac telemetry, and echocardiography. Even after exhaustive searches, no cause for stroke is found in nearly $25 \%$ of cases, leading to a recent focus on determining why these so-called cryptogenic strokes happen and how to prevent their recurrence. ${ }^{1,2}$

Echocardiography is commonly obtained in most patients with ischemic stroke, but its yield is probably modest at best. Although transesophageal echocardiography (TEE) may be superior to transthoracic echocardiography (TTE) for determining an etiology of stroke, whether these findings substantially change management remains debatable. ${ }^{3,4}$ In this issue of the Journal of Hospital Medicine, Marino and colleagues examined the yield of TEE in patients without a known cause of ischemic stroke following a normal TTE. ${ }^{5}$ A possible cause of stroke was identified in $42 \%$, including aortic plaques and patent foramen ovale (PFO), but in only 1 patient did this discovery change management.

Secondary prevention strategies in ischemic stroke outside of atrial fibrillation now almost exclusively involve antiplatelet medications. ${ }^{6}$ Studies of secondary prevention in aortic arch atheromas, patients with depressed systolic function, and those with PFO have failed to demonstrate any strategy that is superior to antiplatelets, and therefore the bar is high to show that any TEE findings impact treatment other than obvious and rare "smoking guns" such as a rare valvular lesion, cardiac tumor, or atrial thrombus. ${ }^{7-9}$

What is more of a recent headline in stroke workup is the increasing emphasis on long-term cardiac monitoring following discharge to detect those with atrial fibrillation, which likely comprise between $15 \%$ and $20 \%$ of cryptogenic stroke patients. ${ }^{10}$ Finding atrial fibrillation clearly changes management and therefore

*Address for correspondence and reprint requests: S. Andrew Josephson, MD, Department of Neurology, Box 0114, 505 Parnassus Avenue, M-798, San Francisco, CA 94143-0114; Telephone: 415-476-9211;

Fax: 415-476-8705; E-mail: andrew.josephson@ucsf.edu

Received: August 23, 2015; Accepted: August 26, 2015 2015 Society of Hospital Medicine DOI 10.1002/jhm.2482

Published online in Wiley Online Library (Wileyonlinelibrary.com). has a higher yield than the vast majority of possible findings on echocardiography. Perhaps in patients in whom a TEE is being considered, extended monitoring should happen first as an outpatient, followed by TEE if the stroke etiology remains obscure. On the other hand, severe left atrial enlargement, thrombus in the atrium, or atrial spontaneous echo contrast ("smoke") are features on echocardiography that might raise the suspicion of atrial fibrillation so high that anticoagulation could be considered while longterm monitoring is being used to definitively prove an atrial arrhythmia.

The current study does have some limitations other than those inherent to its retrospective design. Patients were only included if they were older than 50 years. Some have advocated using TEE as the echocardiogram of choice in young stroke patients due to its perhaps higher yield in these individuals; this study does not address this strategy. At institutions such as ours, an abnormal TTE in a cryptogenic stroke patient is followed by a TEE, and this study again does not alter this approach, because only those with a normal TTE were included. The definition of a normal TTE used in the study was so narrow, including normal left ventricular systolic function, that a majority of stroke patients with vascular risk factors such as hypertension would have likely been excluded. Determining what features and quality of a TTE are so definitive that a TEE is not necessary will be an important thrust of additional research. However, because TEE shows a better view of the left atrial appendage, the aortic arch, and is probably a better shunt study compared with TTE, it is not clear if a normal TTE will ever be adequate to prevent this second more invasive study in selected patients.

At the heart of the matter for health systems is the cost-effectiveness of any screening approach used to determine the etiology of acute ischemic stroke. Studies are underway that will likely demonstrate that long-term monitoring for atrial fibrillation will be worth it. Although it is dubious that TEE would ever fall into the same category due to its low yield, one might imagine a scenario, as our workup for cryptogenic stroke becomes more and more complicated, where obtaining a TEE is cost-effective simply because it gives an answer and therefore can halt further testing. Perhaps at the end of the day, a TEE will just allow us to say to our stroke patients that enough is enough. 
Disclosures: Dr. Josephson receives personal compensation as Editor-inChief of the New England Journal of Medicine Journal Watch Neurology and in an editorial capacity for Continuum Audio.

\section{References}

1. Hart RG, Diener HC, Coutts SB, et al. Embolic strokes of undetermined source: the case for a new clinical construct. Lancet Neurol. 2014;13:429-438.

2. Lin L, Yiin GS, Geraghty OC, et al. Incidence, outcome, risk factors, and long-term prognosis of cryptogenic transient ischaemic attack and ischaemic stroke: a population-based study. Lancet Neurol. 2015;14: 903-913.

3. de Bruijn SF, Agema WR, Lammers GJ, et al. Transesophageal echocardiography is superior to transthoracic echocardiography in management of patients of any age with transient ischemic attack or stroke. Stroke. 2006;37:2531-2534.

4. McGrath ER, Paikin JS, Motlagh B, Salehian O, Kapral MK, O’Donnell MJ. Transesophageal echocardiography in patients with cryptogenic ischemic stroke: a systematic review. Am Heart J. 2014;168:706-712.
5. Marino B, Jaiswal A, Goldbarg S, Bernardini GL, Kerwin T. Impact of transesophageal echocardiography on clinical management of patients over age 50 with cryptogenic stroke and normal transthoracic echocardiogram. J Hosp Med. 2016;11(2):95-98.

6. Kernan WN, Ovbiagele B, Black HR, et al. Guidelines for the prevention of stroke in patients with stroke and transient ischemic attack: a guideline for healthcare professionals from the American Heart Association/American Stroke Association. Stroke. 2014;45:21602236.

7. Amarenco P, Davis S, Jones EF, et al. Clopidogrel plus aspirin versus warfarin in patients with stroke and aortic arch plaques. Stroke. 2014; $45: 1248-1257$.

8. Homma S, Thompson JL, Pullicino PM, et al. Warfarin and aspirin in patients with heart failure and sinus rhythm. N Engl J Med. 2012;366: 1859-1869.

9. Furlan AJ, Reisman M, Massaro J, et al. Closure or medical therapy for cryptogenic stroke with patent foramen ovale. N Engl J Med. 2012;366:991-999.

10. Gladstone DJ, Spring M, Dorian P, et al. Atrial fibrillation in patients with cryptogenic stroke. N Engl J Med. 2014;370:24672477. 